\title{
Control of secondary flow structures on a highly loaded compressor cascade
}

\author{
V Zander and W Nitsche
}

\begin{abstract}
This paper presents detailed flow-field measurements for a compressor cascade equipped with synthetic jet actuators for active flow control. The synthetic jets are mounted on the cascade sidewall and the suction side surface of the blade to reduce the total pressure loss caused by strong secondary flow structures developing in the passage. There are certain articles reporting that synthetic jets are well suited for flow control applications even in axial compressors and cascades. Most of them are focused on the parameter variation to optimize the efficiency of the control approach, still very little is known on the interaction of the synthetic jet actuators with the flow field. Detailed $\mathrm{x}$-wire and pressure measurements were conducted to understand how synthetic jets influence the flow field and what causes the significant loss reduction in the cascade wake. It seems that the added momentum is not the key parameter for flow control with synthetic jets. In fact, the high mixing and the unsteadiness of the jets seem to amplify existing velocity fluctuations in the flow field. These increased fluctuations result in a shift of the shear layer between the flow separation and the surrounding flow, and thus in a dethrottling of the compressor cascade. Together with the increased mixing, loss reductions of approximately $10 \%$ can be reached using synthetic jet actuators.
\end{abstract}

\author{
Keywords \\ Compressors, flow control, synthetic jet actuators, flow-field measurements
}

Date received: 4 June 2013; accepted: 5 June 2013

\section{Introduction}

Modern gas turbine developments tend to further increase the power density of gas turbine engines with the aim to reduce the weight and thus the operational coasts of the engine. The compressor is the major part affecting the weight and size of a turbo engine. Reducing the size and weight of a compressor means to increase the blade loading to achieve the same pressure ratio with a reduced number of compressor stages. The difficulty is to avoid flow separation and thus pressure losses during the pressure build-up and the associated deceleration of the flow field. Therefore, turbo engine compressors are highly optimized parts, ${ }^{1}$ which usually use passive flow control techniques to reduce secondary flow structures that cause the main part of the loss production in compressors. $^{2}$ For example, Hergt et al. ${ }^{3}$ present an advanced endwall contouring method to reduce the pressure losses caused by corner separation in a compressor cascade. Besides the passive techniques, more and more active flow control methods come into the focus of turbomachinery researchers. ${ }^{4}$ An overview of flow control methods can be found in Gad-el Hak. ${ }^{5}$ Furthermore, Cattafesta and Sheplak ${ }^{6}$ summarize the common actuator technology used for active flow control. Some of these concepts and actuators have been tested in compressors as well. A common approach uses compressed air blown out of the surface to avoid flow separation, as approved for example by Nerger et al. ${ }^{7}$ The consumption of bleed air needed to drive the flow control system can be significantly reduced with oscillatory flow control approaches. ${ }^{8}$ An overview of extensive investigations for different unsteady control approaches can be found in Gmelin et al. ${ }^{9}$ and Braunscheidel et al. ${ }^{10}$ Besides pulsed blowing, synthetic jet actuators (SJAs) are used for secondary flow control in compressor cascades. Synthetic jets are zero net mass flux actuators which generate a jet by sucking the ambient fluid into an actuator chamber. In the

Department of Aeronautics and Astronautics, Technical University Berlin, Berlin, Germany

\section{Corresponding author:}

$\checkmark$ Zander, Department of Aeronautics and Astronautics, Technical University Berlin, Berlin 10587, Germany.

Email: vincent.zander@ilr.tu-berlin.de 
subsequent blowing phase, this fluid will be injected back to the flow field as an energized jet. ${ }^{11}$ With this technique it is possible to reenergize a flow that is close to separation without the need for additional high pressure bleed air. The unsteady nature of the synthetic jets and the ring vortices that were formed at the actuator outlet during the blowing phase increase the mixing of the ambient flow field. As a result of the studies presented by Gmelin et al. ${ }^{9}$ it was found that synthetic jets are highly efficient in reducing the pressure losses in a compressor cascade. This was also observed by Matejka et al. ${ }^{12}$ and Zheng et al. ${ }^{13}$ The current paper focuses on the interaction of the SJAs with the cascade flow field. Detailed measurements inside the cascade passage were conducted to clarify the flow control mechanism that forms the basis for flow control with SJAs.

\section{Experimental setup}

The flow control experiments were conducted at the low speed cascade test facility at the Department of Aeronautics and Astronautics at the Technische Universität Berlin. A sketch of the test section is shown in Figure 1. For detailed flow-field investigations, the cascade is built of large-scale profiles with a chord length of $L=0.375 \mathrm{~m}$ and a blade height of $0.3 \mathrm{~m}$. Figure 2 shows the profile geometry together with the actuator positions, indicated by the arrows. With an inflow velocity of $u_{0}=24.5 \mathrm{~m} / \mathrm{s}$ and $M a=0.07$, measurements were conducted at a chord based Reynolds number of $R e=600,000$. The inflow angle was held constant at $\beta_{1}=60^{\circ}$. The SJAs were placed inside the cascade sidewalls and the blade suction side of each passage. Figure 3 presents an oil flow visualization for the baseflow together with the actuator setup used for the investigations. The sidewall actuators are placed perpendicular to the profile contour at $S / S_{\max }=14.5 \%$. According to the flow separation, the suction side mounted actuators were

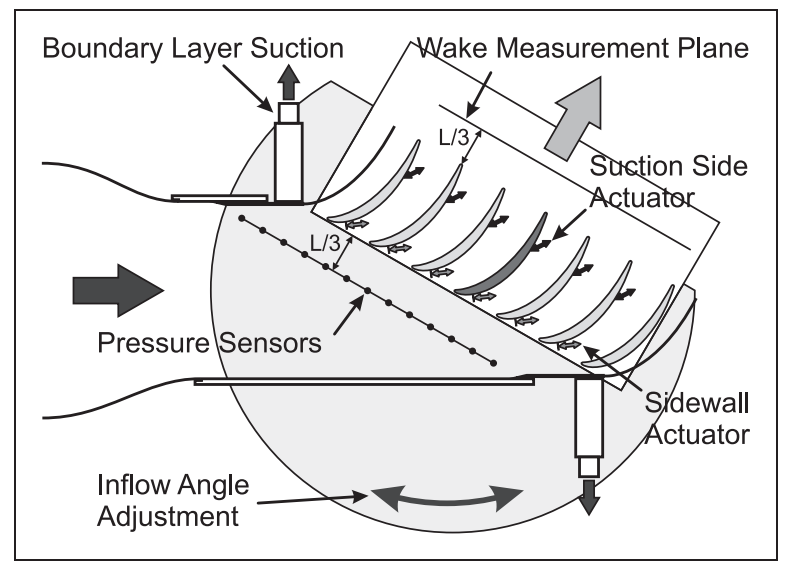

Figure I. Cascade test section. rotated by $45^{\circ}$ to the sidewalls. The center of the actuator slot is placed at $S / S_{\max }=59 \%$ and $T / L= \pm 15 \%$. The slot width is $w=20 \mathrm{~mm}$ for the sidewall actuators, and $35 \mathrm{~mm}$ for the suction side. All actuators have a constant slot depth of $d=0.3 \mathrm{~mm}$. Overall, 24 actuators were mounted to the six flow passages of the cascade test section. The actuators were driven in phase at a constant jet amplitude of $u_{\text {jet }} / u_{1}=1$, and an actuation frequency of $F=230 \mathrm{~Hz}$. For an efficient actuation a jet angle of $\alpha=40^{\circ}$ was used to allow a more tangential blowing. A detailed description of the test section and the flow control setup is given in Zander et al. ${ }^{14}$

Various measurement techniques have been used for the flow-field investigations. Besides the oil flow visualization experiments, surface mounted pressure sensors and hot wires were used to investigate the flow field on the blade suction side. The surface sensors were integrated into a traversable measurement blade to perform measurements over the whole blade height. A more detailed description of the experimental setup is given in Zander et al. ${ }^{15}$ The surface hot wires were calibrated with a skin friction balance

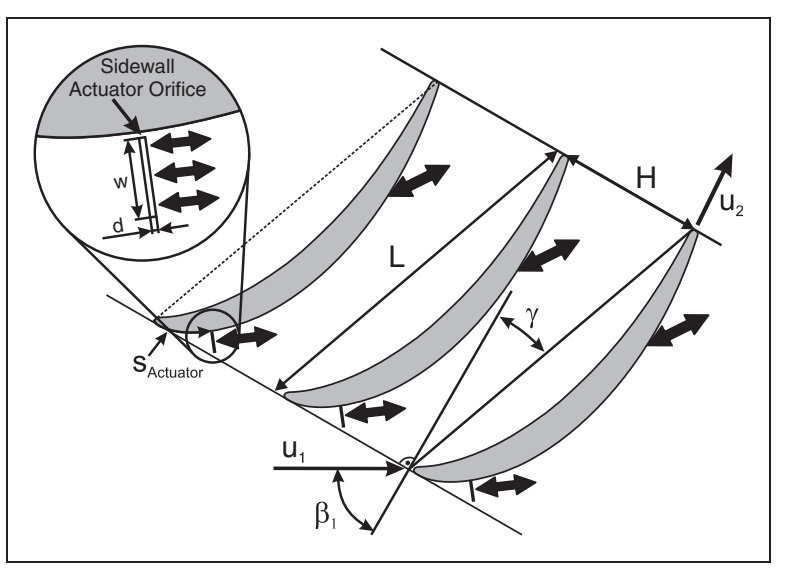

Figure 2. Profile geometry.

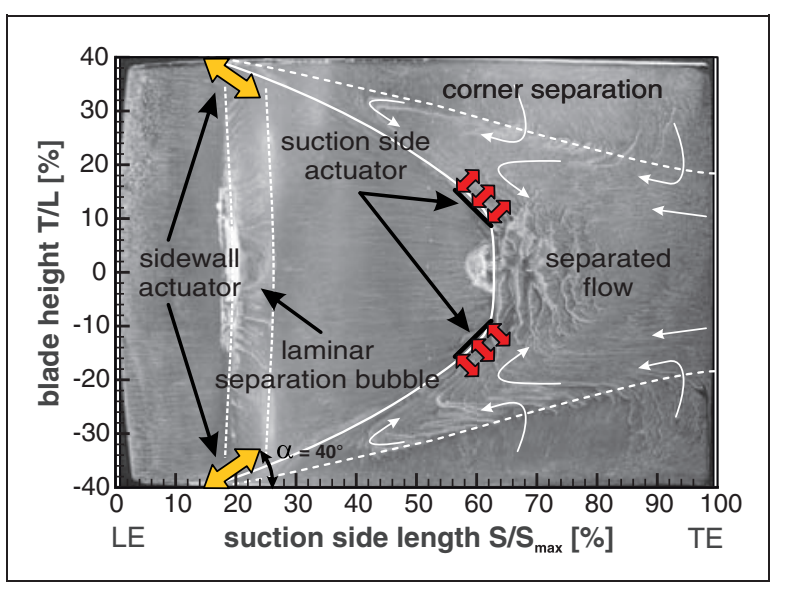

Figure 3. Oil flow visualization and actuator positions. LE: leading edge; TE: trailing edge. 
before they were applied to the cascade. The calibration method is described in Zander et al. ${ }^{16}$ For the flow-field measurements inside the blade passage, a five-hole probe and an $\mathrm{x}$-wire were used. Figures 4 and 5 show the measurement fields for the two different probes used. To gather all three velocity components of the flow field, the $\mathrm{x}$-wire probe was used in two different mounting configurations. Due to the symmetry of the flow field, as can be seen in Figure 3, the probe measurements have only been conducted over a half blade span. The x-wire measurements focused on the interaction of the SJAs with the passage flow field. The high temporal resolution of the $\mathrm{x}$-wire allowed measurements of the turbulent flow fluctuations and a phase averaging of the $\mathrm{x}$ wire signals. The five-hole probe measurements delivered time-averaged values that were used to investigate the cascade wake and to quantify the pressure losses.

\section{Baseflow results}

The measurement techniques used allow a detailed analysis of the cascade flow field. The high aerodynamic blade loading causes the development of strong secondary flow structures on the suction side, as can be seen in the results of the oil flow visualization in Figure 3. The flow is laminar in the

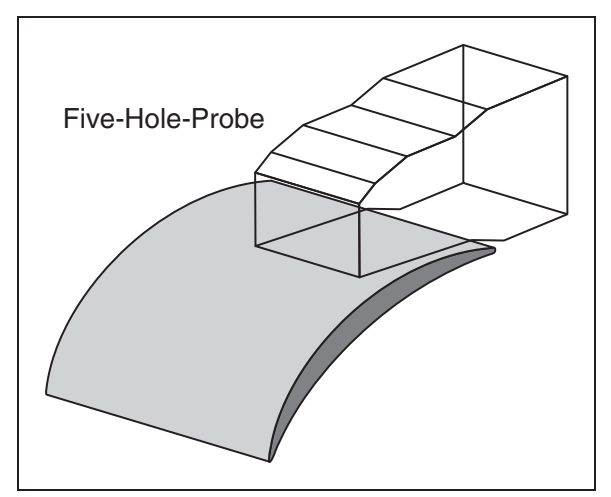

Figure 4. Five-hole probe measurement volume.

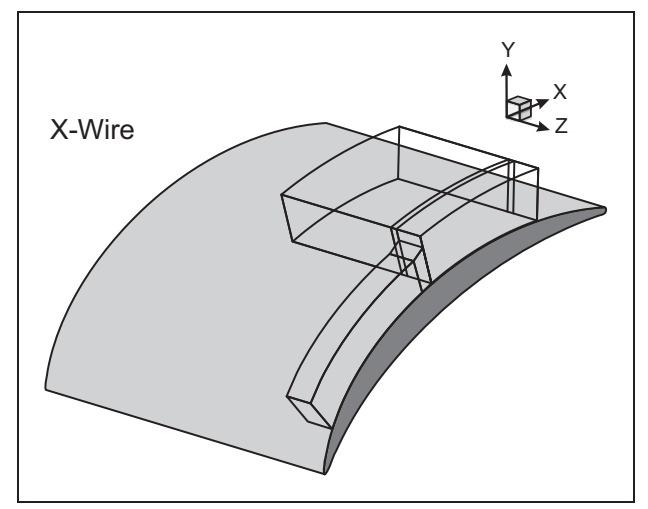

Figure 5. X-wire measurement volume. leading edge (LE) area of the suction side. Transition occurs over a laminar separation bubble at $S / S_{\max }=18 \ldots 24 \%$. After turbulent reattachment, the area of attached flow starts to narrow due to the development of a strong corner stall. Downstream of $S / S_{\max }=62 \%$, the flow is fully separated from the suction side. The strongly three-dimensional flow field required a two-dimensional measurement of the pressure and the wall shear stress distribution. Results at different blade heights are shown in Figures 6 and 7. For the pressure distribution, the common formulation for the pressure coefficient $c_{p}=\left(p-p_{1}\right) / q$ is used. The position of the laminar separation bubble causes a pressure plateau at $X / L=17 \ldots 20 \%$. Downstream of the pressure plateau a strong pressure rise can be observed, which flattens further downstream. The secondary flow structures and the separation have only a minor effect on the pressure distribution, as can be seen by comparing the three different pressure distributions. A separation onset cannot be detected in any of the presented $c_{p}$ distributions. A more detailed view of the secondary flow structures is revealed by the $c_{f}$ distribution on the suction side presented in Figure 7. The wall shear stress is normalized with the dynamic pressure $q$ at the cascade inlet as well. The surface hot wires could not capture the change in flow direction beneath the separation bubble, therefore Figure 7 shows only the magnitude of $c_{f}$. In the LE area, maximum $c_{f}$ values can be detected due to the thin boundary layers in this region. The laminar separation bubble causes a strong decrease in $c_{f}$, followed by a strong rise related to the turbulent reattachment. Downstream of the reattachment, a distinct influence of the secondary flow structures on the $c_{f}$ distribution can be observed. The results at blade midspan show a flow separation, occurring around $X / L=65 \%$, which corresponds to the position of $S / S_{\max }=62 \%$ observed in the oil flow visualization. A similar drop in $c_{f}$ caused by the

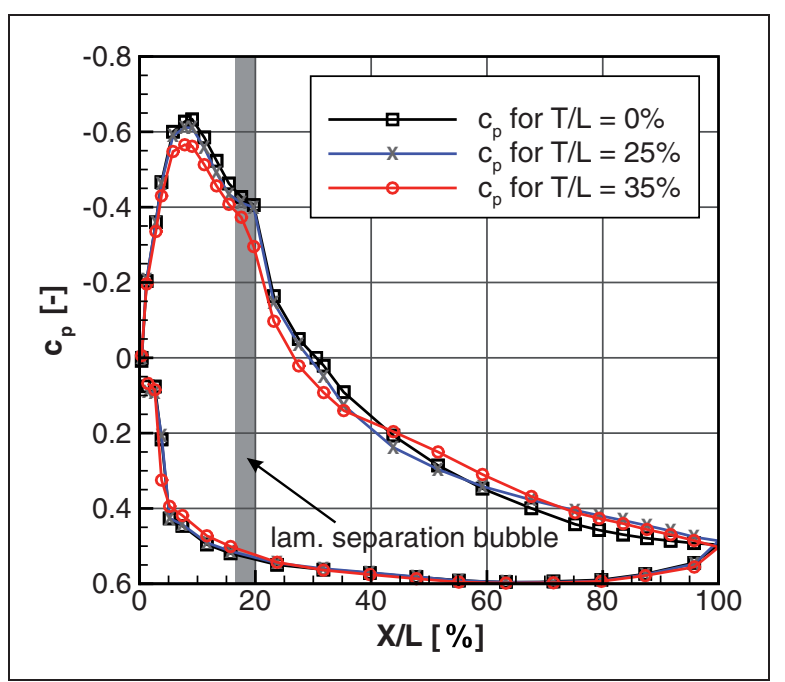

Figure 6. $c_{p}$ distribution. 
corner separation can also be seen for the profile at $T / L=25 \%$. At the measurement position of $T / L=35 \%$ the strong corner vortex dominates the $c_{f}$ distribution, which causes a slight increase in the $c_{f}$ values at $40 \%<X / L<65 \%$. A flow separation is only indicated by the last sensor at $X / L=85 \%$.

Results for five-hole probe measurements in the wake plane $L / 3$ downstream of the trailing edge (TE) are presented in Figure 8. Due to the symmetry of the flow field observed in Figure 3, measurements were only conducted over the half blade high $(37.5 \% \leqslant T / L \leqslant 0)$. For the presentation an area averaging was applied to the axial velocity ratio $u_{2 a x} / u_{1 a x}$ and the static pressure rise $\Delta p=\left(p_{2}-p_{1}\right) / q$, whereas a mass weighted averaging was used for the total pressure loss $\zeta$ and the exit flow angle $\beta_{2}$. The secondary flow structures and the large flow separation cause significant pressure losses in the cascade wake, whereas there is only a minor influence on the static pressure distribution. The $\Delta p$

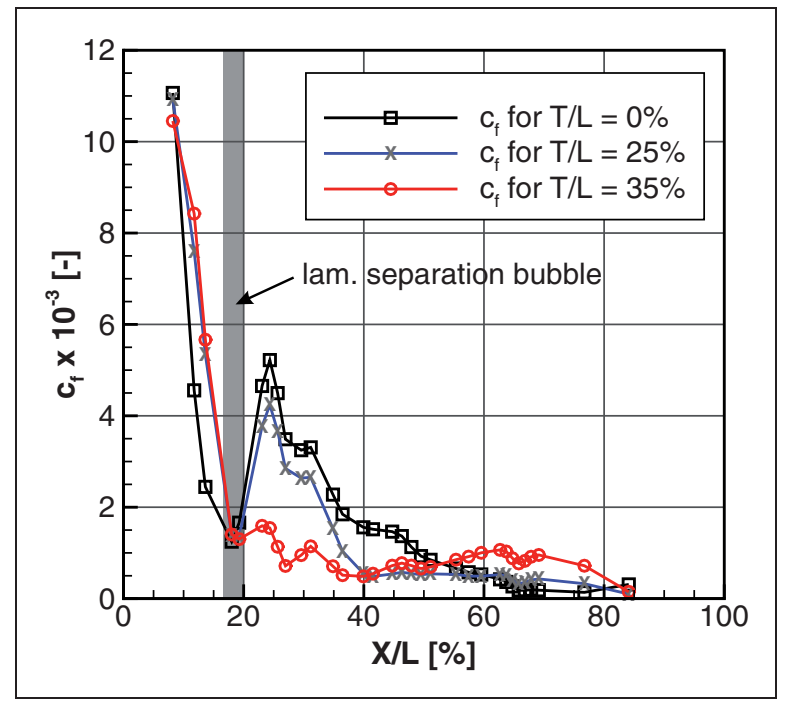

Figure 7. $c_{f}$ distribution. distribution is nearly uniform and rises only slightly in the vicinity of the sidewall. In contrast to this, the mean axial velocity ratio shows a distinct variation over the blade height. $u_{2 a x} / u_{1 a x}$ shows an inverse trend compared to the total pressure loss, and is, besides the total pressure loss, the most significant wake parameter in the case of synthetic jet actuation. The exit flow angle shows the typical trend for compressor cascades. Due to the secondary flows an over-turning occurs close to the sidewall and a slight under-turning of $\beta_{2}=1.5^{\circ}$ at blade midspan.

A more detailed picture of the flow field can be given by analyzing the $\mathrm{x}$-wire measurements inside the blade passage, presented in Figures 9 and 10 . Both show the flow field on the blade suction side for one half of the blade. The left figure shows the

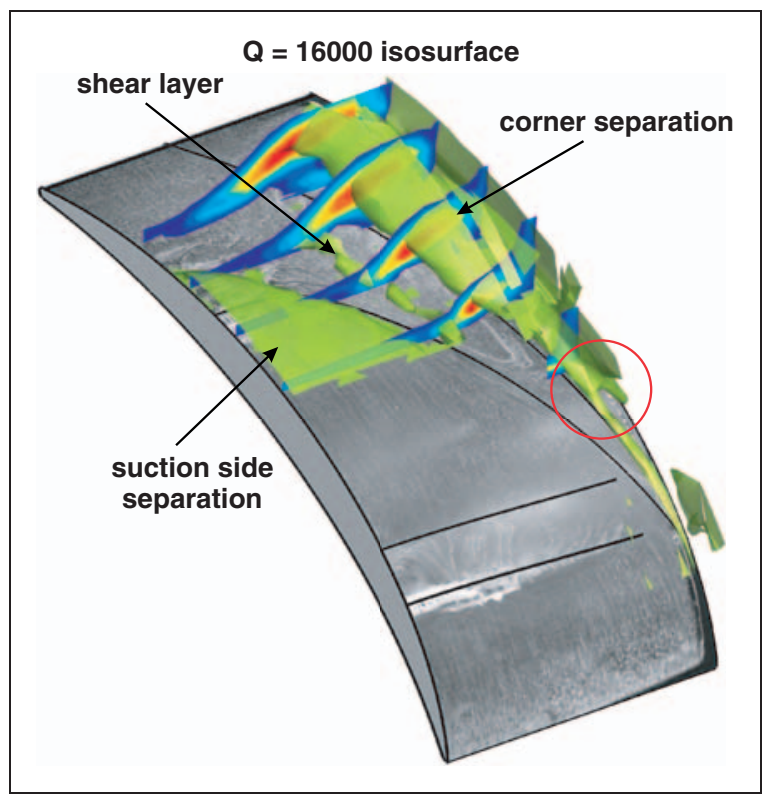

Figure 9. $Q$ isosurface and turbulent kinetic energy slices on the suction side.
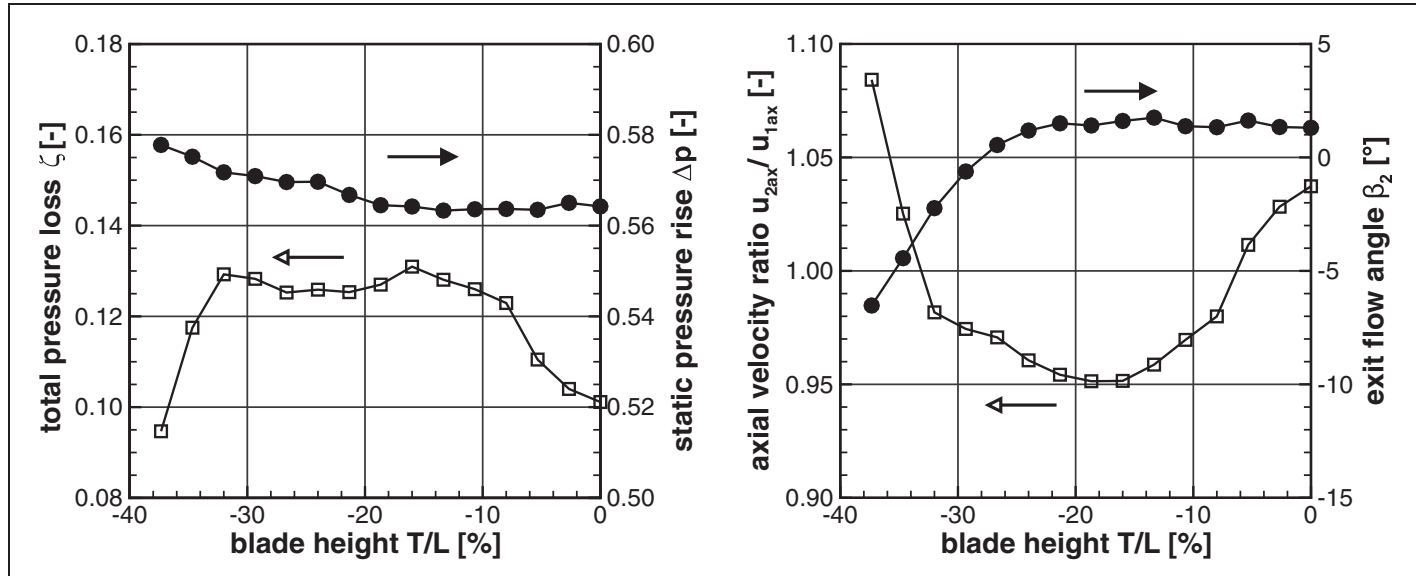

Figure 8. Wake characteristics for the baseflow. 
oil flow visualization picture on the suction side together with an isosurface of the $Q$-criteria calculated out of the measured velocity field. Furthermore, five turbulent kinetic energy (TKE) slices are plotted for different axial positions. Positive values of the $Q$-criteria are well suited for the detection of vortex structures in flow fields. ${ }^{17}$ The result for $Q=16,000$ clearly shows the corner separation close to the sidewall. Furthermore, the shear layer of the flow separation at blade midspan and a second shear layer between the corner vortex and the flow separation are visualized by the isosurface. The results of the $Q$-criteria and the oil flow visualization show a good agreement and reveal a detailed view of the dominating flow structures on the suction side. It can be seen that there is a sudden vortex burst (marked with the circle) downstream of the laminar separation bubble, which is responsible for the strong passage blockage and the high pressure losses. The TKE slices show maximum values between the corner separation and the flow field at midspan. This indicates a strong interaction of the corner stall with the flow separation at midspan. Figure 10 presents the TKE slices together with the measured $R M S\left(c_{p}\right)$ distribution on the blade suction side. The pressure fluctuations reveal a similar picture of the suction side flow as the oil flow visualization. At the position of turbulent reattachment, maximum $R M S\left(c_{p^{\prime}}\right)$ can be found on the blade surface. Furthermore, there are strong pressure fluctuations in the region between the corner stall and the separation around blade midspan. The higher $R M S\left(c_{p^{\prime}}\right)$ in this region corresponds to the increased velocity fluctuations of the flow field in this part of the suction side shown by the TKE slices.

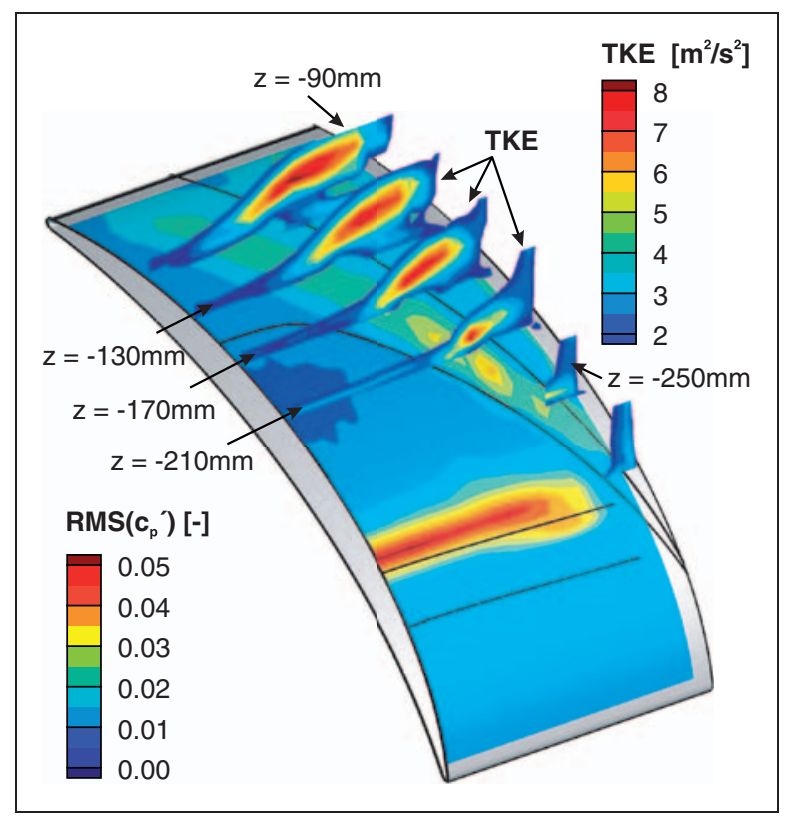

Figure 10. Velocity and pressure fluctuations on the suction side.

TKE: turbulent kinetic energy.
With the SJAs it was attempted to influence the secondary flow structures to reduce the corresponding flow losses. The used SJAs add only a small amount of momentum to the flow field, due to their small jet velocity ratios and the narrow slot sizes. Significant loss reductions can be obtained by means of an increased mixing of the flow inside the passage.

\section{Flow control results}

A wide parameter study has been carried out for the flow control approach used in these experiments. A detailed description of the results and an efficiency consideration is given in Zander et al. ${ }^{14}$ Numerical investigations concerning these measurements were published in Gmelin et al., ${ }^{18}$ whereas this report focuses on the flow effects caused by the synthetic jet actuation to reduce the pressure losses of the cascade. The flow control parameters presented in the description of the experimental setup were used for all measurements presented here. Wake measurement results for the baseflow and the actuated flow field can be seen in Figure 11. For the analysis of the wake characteristics, only a comparison of the total pressure loss and the axial velocity ratio is given, because the static pressure rise and the exit flow angle did not change in the case of flow control. On the left side of Figure 11, it can be seen that the total pressure loss reduces over the entire blade height in the case of actuation. Especially between $T / L=-30 \%$ and $T / L=-10 \%$, a nearly uniform loss reduction can be observed. But there is also a loss reduction at midspan, although the synthetic jets are placed in the sidewall and around $T / L=-15 \%$ only. These results indicate that the flow control effect of the synthetic jets is not limited to the area nearby. Instead, they affect the whole flow field. Besides the total pressure loss, minor changes can also be seen in the distribution of the axial velocity ratio. The major changes occur around blade midspan. Due to the actuation, $u_{2 a x} / u_{1 a x}$ rises, which indicates a higher mass flow through the center of the blade. Because of the mass weighted averaging applied to the total pressure loss, a part of the loss reductions is caused by the dethrottling of the cascade. This effect is particularly distinct at blade midspan. Although a mass weighted averaging is applied to the exit flow angle (not shown here), no changes can be seen for this wake parameter. Furthermore, the static pressure rise did not change. This can also be observed in the comparison of the $c_{p}$ distribution measured at blade midspan, presented in Figure 12. The static pressure distribution is not affected by the synthetic jet actuation. Especially the TE pressure did not change, which shows that the static pressure distribution of the cascade wake is not influenced by the actuation. This indicates that the exit flow conditions of the cascade remain constant, which includes the exit flow angle as well. In contrast to this, Figure 13 shows that there is a 


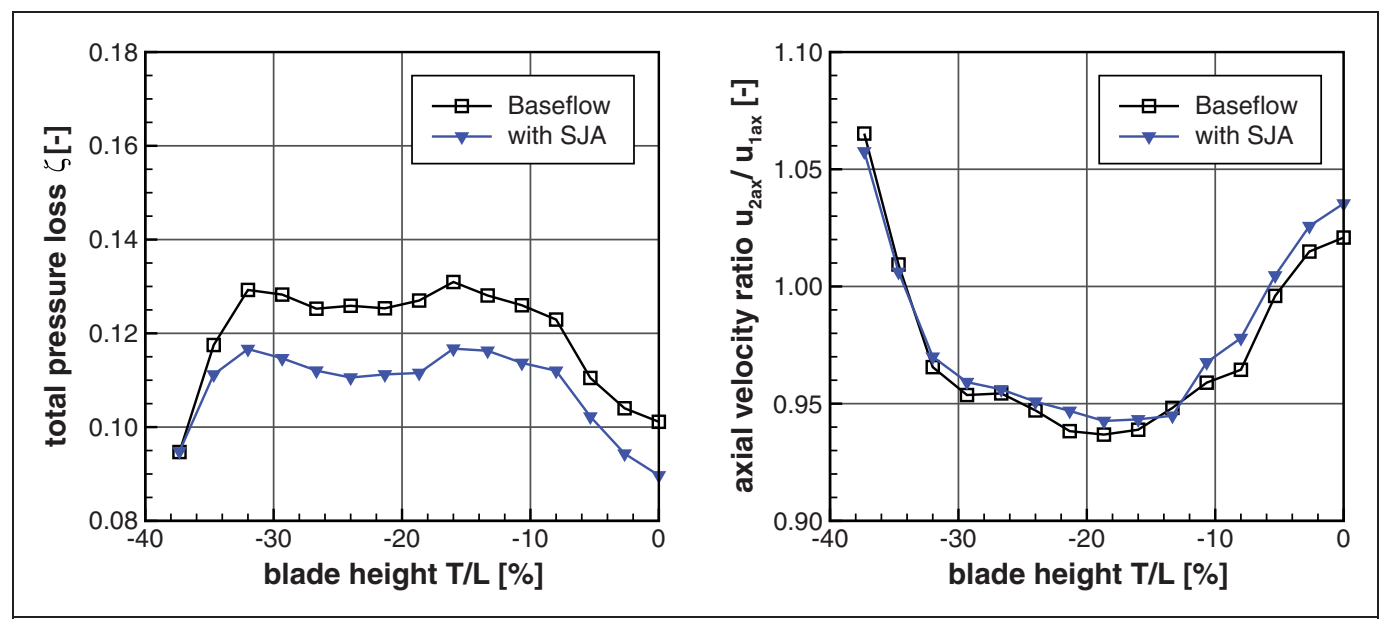

Figure II. Pressure loss and velocity ratio in the case of flow control. SJA: synthetic jet actuator.

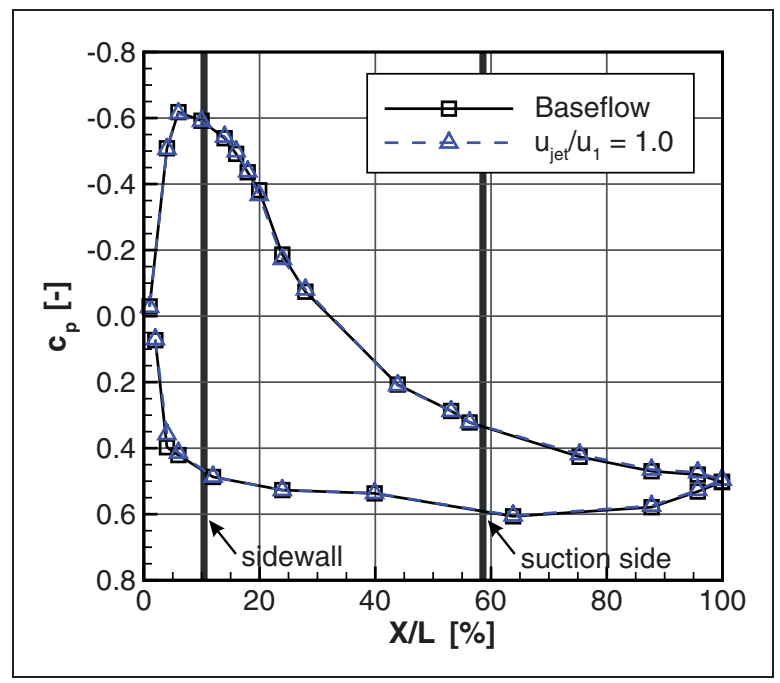

Figure 12. $c_{p}$ distribution with and without actuation.

significant influence of the synthetic jets on the pressure fluctuations at midspan. In the case of actuation, the $\operatorname{RMS}\left(c_{p^{\prime}}\right)$ values increase over the whole blade surface and show a strong dependency on the jet amplitude, except at the position of the turbulent reattachment. At this position a slight decrease in the pressure fluctuations can be observed. The measurements indicate that the SJAs amplify the fluctuations of the whole flow field, not only in the vicinity of the actuators. This amplification effect is also strongly frequency dependent and is discussed in more detail in Zander et al. ${ }^{14}$

Deeper insight into the flow field is provided by the $\mathrm{x}$-wire measurements within the blade passage. These measurements help to find the main flow features that cause the loss reductions in the case of flow control and thus understanding the flow control mechanism of synthetic jet actuation in complex flow fields. As a result of the pressure measurements, which show no

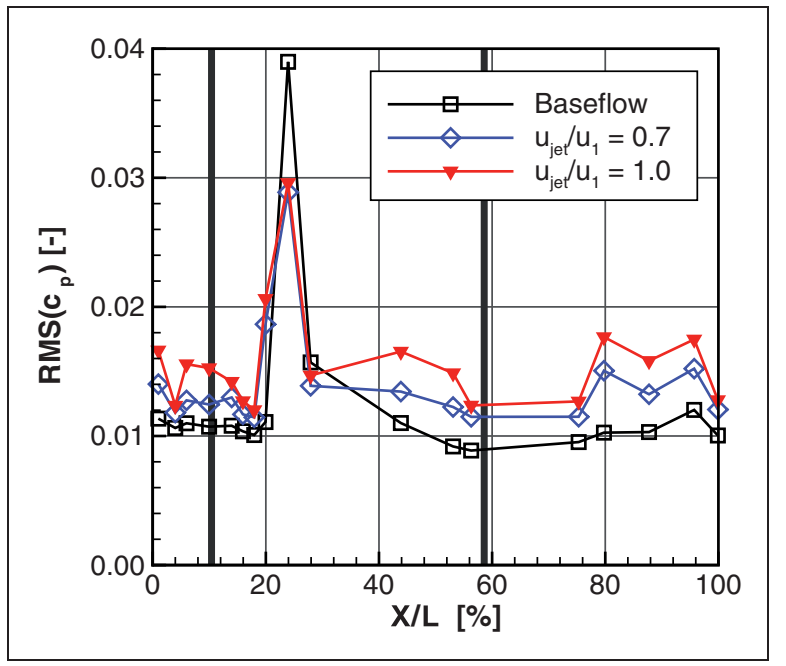

Figure 13. Pressure fluctuations at midspan for different jet amplitudes.

significant changes in the mean flow field, the analysis of the $\mathrm{x}$-wire measurements focuses on the velocity fluctuations. The measurements give the possibility to calculate all terms of the Reynolds stress tensor, except the product of the two cross-flow velocity components $v /$ and $w \%$. A detailed analysis of the velocity fluctuations reveals that the term $\overline{u^{\prime}}$ is twice as large as the other fluctuation products and therefore dominates the turbulent energy exchange inside the passage. Figure 14 shows $\Delta \overline{u^{\prime 2}}=\overline{u_{S J A^{\prime}}{ }^{2}}-\overline{u_{0^{\prime}}{ }^{2}}$ on different slices, which points out the changes of the velocity fluctuations caused by the synthetic jet actuation. Positive values indicate an increase in velocity fluctuations, whereas negative values represent a reduction in the case of flow control. The suction side surface shows an oil flow visualization of the actuated flow field. Furthermore, the position of the SJAs is shown by red lines on the sidewall and the suction side surface. Compared to the visualization of the baseflow, 


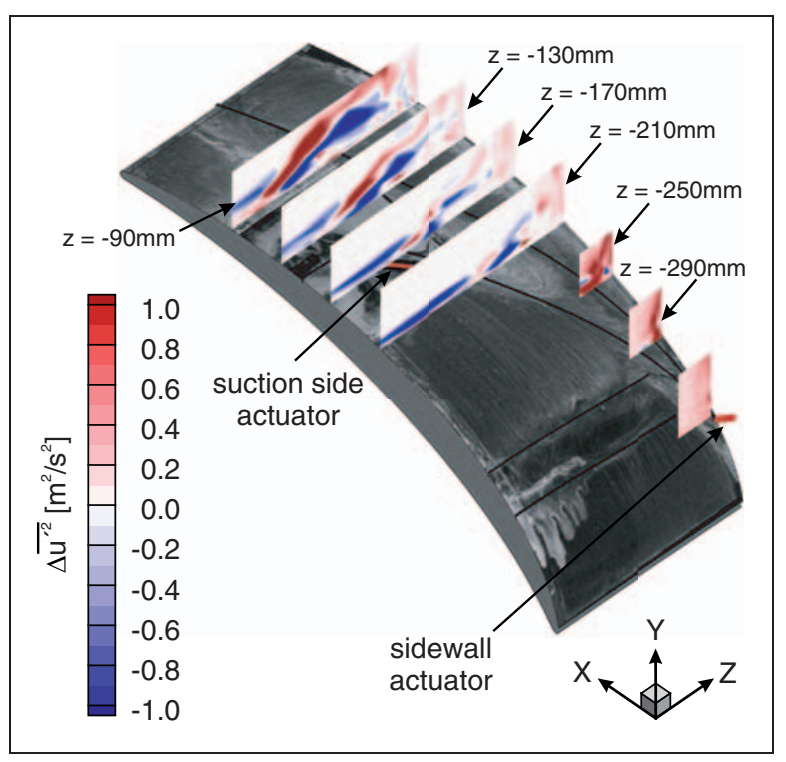

Figure 14. $\Delta \overline{u^{\prime 2}}$ change due to actuation and oil flow visualization for the actuated flow field.

no significant changes can be found in the flow topology close to the surface, as it was seen in the pressure distribution. This can also be confirmed by comparing the mean velocity fields with and without actuation (not shown here). This leads to the conclusion that the mean flow field shows only minor changes when the SJAs were used for flow control, and that the major control effect is caused by the increased fluctuation amplitudes. Figure 14 shows a significant rearrangement of the turbulent velocity fluctuations. A distinct increase in $\overline{u^{2}}$ can be detected downstream of the sidewall actuator. The results show downstream of $z=-210 \mathrm{~mm}$ regions with reduced velocity fluctuations around midspan. The reduction at blade midspan explains the increased mass flow rate in this part of the blade as observed in the wake characteristics in Figure 11. The reduced velocity fluctuations in the area of the corner stall denote a displacement of the shear layer that forms between the corner vortex and the separation at blade midspan. Higher values of $\overline{u^{2}}$ in the upper part of the observed flow region indicate a shift of the shear layer as shown in Figure 15. The shear layer is forced closer to the surface at midspan, whereas it moves away from the surface at $T / L=-20 \ldots-30 \%$. This shift dethrottles the midspan region and causes an increased blockage in the region of the corner stall. The results of the five-hole probe measurements inside the flow passage verify this modification of the flow field. Figure 16 shows the difference in the total pressure loss $\Delta \zeta=\zeta_{\text {SJA }}-\zeta_{0}$. Reduced losses are indicated by negative values and increased loss values by positive differences. The loss difference shows the same shift as observed for the velocity fluctuations. Distinct loss reductions appear close to the blade surface and especially at midspan region. In the region of the corner vortex, slightly increased loss values can be

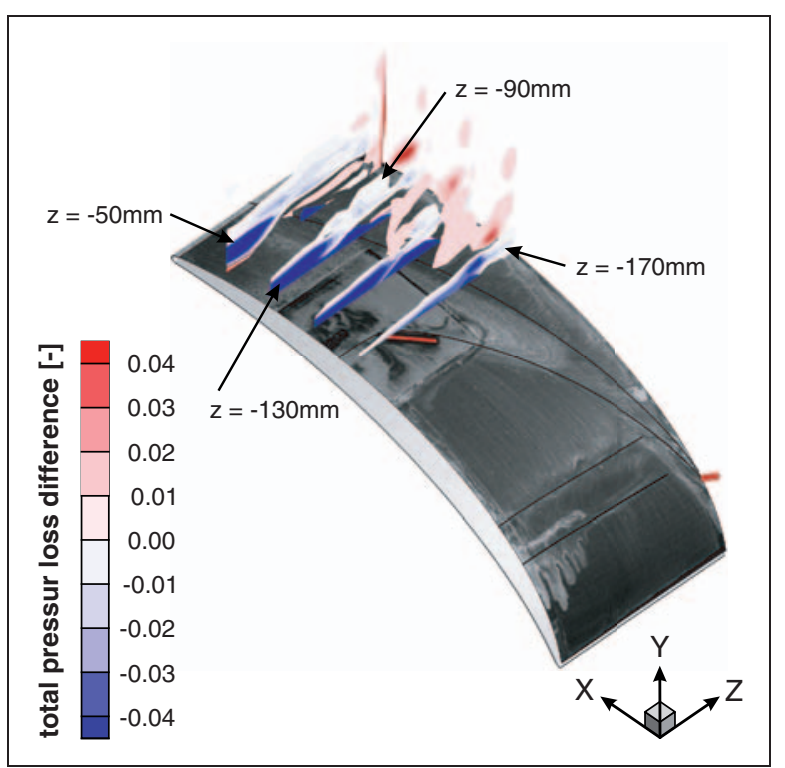

Figure I5. Total pressure loss difference $\zeta_{\text {SJA }}-\zeta_{0}$.

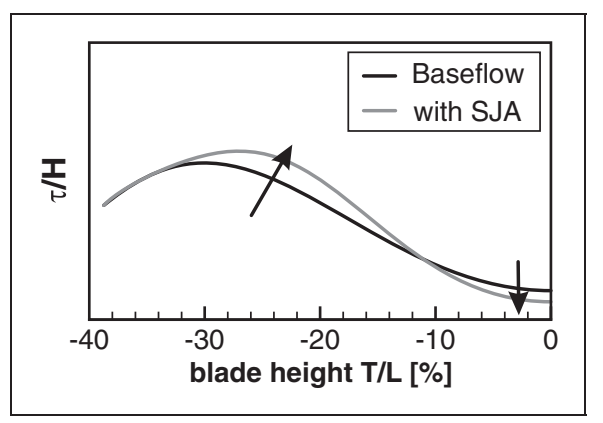

Figure 16. Shear layer shift.

SJA: synthetic jet actuator.

detected. They are caused by the upward shift of the corner vortex.

The flow field in the passage shows a shift of the shear layer between the corner stall and the separation around midspan, which causes decreased losses at the blade center and slightly higher loss values in the region of the corner stall. A different picture was observed analyzing the wake measurement results in Figure 11. They show a nearly uniform loss reduction over the whole blade height. Therefore, Figure 17 presents a comparison of the TKE in the wake of the cascade together with the total pressure loss isolines. The presented results show clear loss reductions in the zones with the highest pressure losses $\zeta \geqslant 0.4$. These reductions extend over a wide range of $T / L$ as seen in Figure 11, whereas the absolute size of the loss region with $\zeta=0.1$ did not change in the case of actuation.

A comparison of the TKE distribution in the cascade wake shows that maximum TKE values can be found in the regions with high pressure loss gradients. The $T K E$ level rises in these regions in the case of flow control using SJAs. Increasing values can be observed 


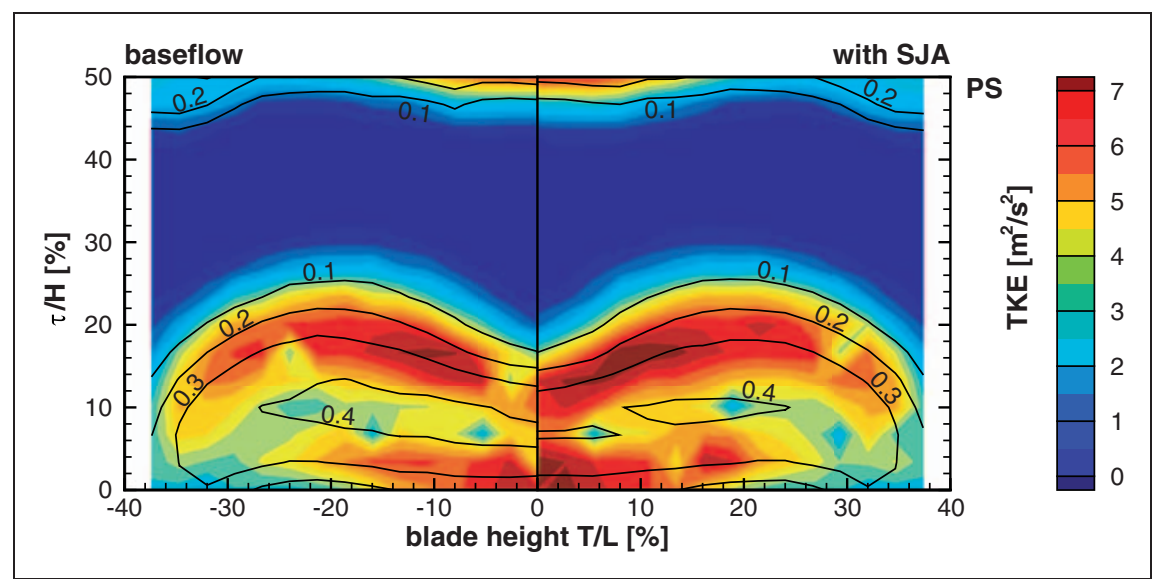

Figure 17. TKE and pressure loss in the wake of the cascade. TKE: turbulent kinetic energy; SJA: synthetic jet actuator.

especially around $T / L=0$. The higher turbulence level in this region causes a stronger mixing of the flow and thus a redistribution of the pressure losses. The slightly increased loss values that can be observed inside the passage were mixed out in the wake and vanish in the wake measurement plane. In conjunction with the higher passage mass flow, this results in a loss reduction of nearly $10 \%$ compared to the baseflow. The synthetic jets amplify the velocity fluctuations inside the shear layer between the corner separation and the flow at midspan. In this way, the spent energy for the actuation can be more than compensated by the loss reductions in the cascade wake ${ }^{14}$

\section{Conclusions}

The detailed measurement results presented in this paper give deeper insight into the interaction of SJAs with the cascade flow and allow an analysis of the loss reduction achieved with the flow control approach. Pressure measurements on the blade surface and $\mathrm{x}$-wire measurements above the suction side show that the fluctuations rise in the case of actuation, whereas the mean flow field shows only minor changes. Furthermore, it is evident that the actuator signal is amplified by the flow field which can cause loss reductions of approximately $10 \%$ exceeding the amount of energy spent for driving the SJAs. The analysis of the baseflow configuration reveals a shear layer with high TKE that forms between the corner stall and the flow separation at blade midspan. With the SJAs mounted on the cascade sidewall and the blade suction side it is possible to deform this shear layer and to enhance the turbulent velocity fluctuations in the cascade wake. Shifting the shear layer close to the blade surface at midspan dethrottles the cascade and causes a higher mass flow rate in this part of the blade. The increased fluctuations cause a stronger mixing in the wake of the cascade. These two effects results in the significant loss reductions. Previous investigations have shown that the control results depend on the forcing frequency and the jet amplitude. Using natural instabilities of the flow field for actuation results in an amplification of the dominant flow fluctuations, which can lead to significant changes in the loss production. As discussed in a previous article, the amplitude of the forcing signal has to exceed a threshold minimum value of $u_{j e t} / u_{1}>0.8$ to reveal positive control effects, but can still be limited to practical values for real turbomachines of $u_{\text {jet }} / u_{1} \approx 1 .^{14}$

\section{Funding}

This research received no specific grant from any funding agency in the public, commercial, or not-for-profit sectors.

\section{References}

1. Wennerstrom AJ. Highly loaded axial flow compressors: history and current developments. ASME J Turbomach 1990; 112: 567-578.

2. Denton J. Loss mechanisms in turbomachines. J Turbomach 1993; 115: 621-656.

3. Hergt A, Dorfner C, Steinert W, et al. Advanced nonaxisymmetric endwall contouring for axial compressors by generating an aerodynamic separator, part ii: experimental and numerical cascade investigation. J Turbomach 133: 8-2011.

4. Lord WK, MacMartin DG and Tillman TG, Flow control opportunities in gas turbine engines. In Fluids 2000 , Denver, 2000, paper no. AIAA 2000-2234.

5. Gad-el Hak M. Modern developments in flow control. Appl Mech Rev 1996; 49: 365-379.

6. Cattafesta III L and Sheplak M. Actuators for active flow control. Annu Rev Fluid Mech 2010; 43: 247-272.

7. Nerger D, Saathoff H, Radespiel R, et al. Experimental investigation of endwall and suction side blowing in a highly loaded compressor stator cascade. In: Proceedings of ASME turbo expo 2010: power for land, sea and air, Glasgow, UK, 2010, paper no. GT201022578. 
8. Seifert A, Bachar T, Koss D, et al. Oscillatory blowing: a tool to delay boundary-layer separation. AIAA J 1993; 31: 2052-2060.

9. Gmelin C, Zander V, Hecklau M, et al. Active flow control concepts on a highly loaded subsonic compressor cascade: résumé of experimental and numerical results. In: $A S M E$ turbo expo 2011, Vancouver, Canada, 2011, paper no. GT2011-46468.

10. Braunscheidel EP, Culley DE, and Zaman KB. Application of synthetic jets to reduce stator flow separation in a low speed axial compressor. Technical Report AIAA 2008-0602, NASA Glenn Research Center, Cleveland, OH, 2008.

11. Glezer A and Amitay M. Synthetic jets. Annu Rev Fluid Mech 2002; 34: 503-529.

12. Matejka M, Safarik P, Popelka L, et al. Influence of active methods of flow control on compressor blade cascade flow. In: ASME turbo expo, 2008, GT200851109.

13. Zheng X, Zhou S, Lu Y, et al. Flow control of annular compressor cascade by synthetic jets. $J$ Turbomach 2008; 130: 1-7.

14. Zander VF, Hecklau M, Nitsche W, et al. Active flow control by means of synthetic jets on a highly loaded compressor cascade. J Power Energy 2011; 225: 897-906.

15. Zander V, Hecklau M, Nitsche W, et al. Active control of corner vortices on a highly loaded compressor cascade. In 8th European turbomachinery conference, Graz, 2009.

16. Zander V, Dobriloff C, Lumpe M, et al. Wall shear stress measurements on a highly loaded compressor cascade. In ASME turbo expo 2011, Vancouver, Canada, 2011, paper no. GT2011-46364.

17. Hunt J, Wray A and Moin P. Eddies, streams, and convergence zones in turbulent flows. In: Proceedings of the 1988 CTR summer program, vol. 2, 1988, pp. 193-208.

18. Gmelin C, Steger M, Zander V, et al. Numerical investigations of active flow control using synthetic jets on a highly loaded compressor stator cascade. In: Proceedings of ASME 2010 3rd joint US-European fluids engineering summer meeting and 8th international conference on nanochannels, microchannels, and minichannels, Montreal, Canada, 2010.

\section{Appendix}

\section{Notation}

$\begin{array}{ll}c_{f} & \text { skin friction coefficient } \\ c_{p} & \text { pressure coefficient } \\ F & \text { frequency }(1 / \mathrm{s}) \\ H & \text { blade pitch }(\mathrm{m}) \\ L & \text { chord length }(\mathrm{m}) \\ M a & \text { Mach number } \\ p & \text { pressure }(\mathrm{Pa}) \\ q & \text { dynamic pressure }(\mathrm{Pa}) \\ R e & \text { Reynolds number } \\ S & \text { length from the leading edge }(\mathrm{m}) \\ S_{\text {max }} & \text { total length of blade side }(\mathrm{m}) \\ T & \text { blade height }(\mathrm{m}) \\ u, v, w & \text { velocity components }(\mathrm{m} / \mathrm{s}) \\ x, y, z & \text { coordinates }(\mathrm{m}) \\ \alpha & \\ \beta & \text { jet angle }\left(^{\circ}\right) \\ \gamma & \text { flow angle }\left(^{\circ}\right) \\ \zeta & \text { stagger angle }\left({ }^{\circ}\right) \\ \tau & \text { total pressure loss }\end{array}$

\section{Subscripts}

$\begin{array}{ll}0 & \text { baseflow } \\ 1 & \text { inlet } \\ 2 & \text { outlet } \\ s & \text { static } \\ t & \text { total }\end{array}$

